\title{
Niederländische Diabetikerfüße besonders gut betreut
}

Fragestellung: Anhand einer repräsentativen niederländischen Patientengruppe sollte die Inzidenz von diabetischen Fußulzera erhoben werden.

Hintergrund: Aktuelle populationsbezogene Daten zur Häufigkeit von diabetischen Fußulzera sind rar. Die meisten Daten stammen aus den 1990er Jahren. Seither hat die Häufigkeit von Typ-2-Diabetes zugenommen und die Organisation der Diabetesbehandlung hat sich in vielen Ländern geändert.

Patienten und Methoden: Es wurde die NIVEL-(Netherlands Institute for Health Services Research) Primary Care Database genutzt. Diese Datenbasis enthält Daten der standardisierten Routinebehandlung von Niederländern aus 355 Allgemeinpraxen und ist repräsentativ für die niederländische Bevölkerung. In diesem Datensatz fanden sich 81.793 Patienten mit einem Diabetes mellitus. Die jährlichen Inzidenzraten für Fußulzera und andere pathologische Fußbefunde wurden für die Jahre 2010 bis 2013 erhoben. Um Ungenauigkeiten zu vermeiden wurden Inzidenzraten erhoben: 1 . für alle Patienten mit Diabetes, 2. für diejenigen, die eine dokumentierte Fußuntersuchung hatten und 3. für diejenigen Patienten mit dokumentierten Vorhandensein oder Ausschluss einer Fußulzeration.

Ergebnisse: Es fanden sich 412 Patienten mit einem dokumentierten Fußulkus (0,5\%). Eine komplette Fußuntersuchung war bei 46.087 (56 \%) Patienten durchgeführt worden, eine inkomplette Fußuntersuchung bei $64 \%$ der Patienten. Die jährliche Inzidenz von Fußulzera betrug 0,34 \% bei Berücksichtigung derjenigen, die wenigstens

\section{Originalie}

Stoekenbroek RM, Lokin JLC, Nielen MM et al. How common are foot problems among individuals with diabetes?

Diabetic foot ulcers in the Dutch population. Diabetologia. 2017 Jul;60(7):1271-5
densein/Ausschluss einer Fußulzeration, würde die Inzidenz bei $1,08 \%$ liegen. Bei denjenigen Patienten mit dokumentierter Fußuntersuchung fanden sich bei $15 \%$ fehlende Fußpulse, bei 17 $\%$ eine periphere Polyneuropathie und bei $10 \%$ Schwielen oder Druckstellen der Haut.

Schlussfolgerungen: Die Autoren sehen im Vergleich zu bisher erhobenen Daten in den Niederlanden und anderen europäischen Ländern eine geringere Inzidenz von diabetischen Fußulzera. Andererseits erfassten sie eine erhebliche Prozentzahl von ca. $20 \%$ mit Risikofaktoren für eine Fußulzeration. Sie folgern aus ihren Daten, dass möglicherweise die verbesserte Versorgung der Patienten mit Diabetes in den Niederlanden für diese niedrigen Inzidenzen an Fußulzera verantwortlich ist.

\section{- Kommentar von Prof. Dr. med. M. Spraul}

\section{Fortschritte in Versorgungsstrukur senken Risiko}

Diese Ergebnisse sind in der Tat recht positiv, im Vergleich zu den bisher bekannten Zahlen zur Inzidenz von Fußulzerationen, die in den meisten Studien zwischen 1,9 und 2,6\% lagen. Diese Studie ist ein weiterer Mosaikstein, der nachweist, dass eine verbesserte Versorgungsstruktur der Patienten mit Diabetes zu einer Reduzierung des Risikos führt, ein diabetisches Fußsyndrom zu erleiden.

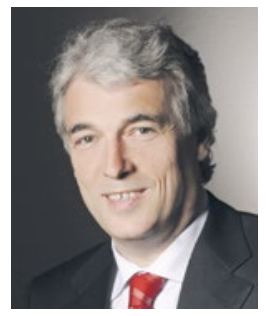

Prof. Dr. med. Maximilian Spraul

Mathias-Spital \& Jakobi-Krankenhaus Interdisziplinäres Diabetes-Fuß-Zentrum Frankenburgstr. 31, 48431 Rheine m.spraul@mathias-spital.de 